\title{
Editorial
}

\section{COVID-19 vaccine}

\author{
MMSU Islam
}

COVID-19 is now a global pandemic, first detected in Wuhan, Hubei province, China, on December 31, 2019 ${ }^{1}$. IEDCR (Institute of Epidemiology Disease Control and Research) declared the first three cases in Bangladesh on $8^{\text {th }}$ March, 2020 ${ }^{2}$. Till today (6 February, 2021) total COVID-19 cases are 105,917,456 and total death are 2,309,058 in the world ${ }^{3}$.

There are no specific and effective treatments, so prevention is the best option and whole world is searching for effective vaccine. Vaccines work by training and preparing the body's immune system; to recognize and fight off the viruses. If the body is exposed to those disease-causing germs later, the body is immediately ready to destroy them, preventing illness ${ }^{4}$.

The process of vaccine development is very long, sometimes take over a decade to complete. In the search for a COVID-19 vaccine, researchers and developers are working on several different phases in parallel, to speed up results. Also, national and international health organizations are working together through COVAX. COVAX is the vaccines pillar of the Access to COVID-19 Tools \& 64 higher income economies have joined the COVAX Facility to invest in development and equitable distribution of vaccines ${ }^{5}$.

As of December 2020, there are over 200 vaccine candidates for COVID-19 being developed. Of these, at least 52 candidate vaccines are in human trials. There are several others currently in phase I/II, which will enter phase III very soon. There are three main approaches to design a vaccine; a). The whole-microbe approach (Inactivated vaccine, Live-attenuated vaccine, Viral vector vaccine) b). The subunit approach and c). The genetic approach (nucleic acid vaccine). Nucleic acid approach is a new way of developing vaccines and just uses a section of genetic material (not the whole microbe) that delivers a specific set of instructions to our cells, either as DNA or mRNA, for them to make the specific protein that we want our immune system to recognize and respond to ${ }^{6}$.

Dr. M. M. Shahin-Ul-Islam, MBBS, FCPS (Medicine), MD (Gastroenterology), Associate Professor (CC), Department of Gastroenterology, Faridpur Medical College, Faridpur.

Address of correspondence :

Dr. M. M. Shahin-Ul-Islam, MBBS, FCPS (Medicine), MD (Gastroenterology), Associate Professor (CC), Department of Gastroenterology, Faridpur Medical College, Faridpur.

Phone:+88-01711986290, E-mail:shahin52dmc@gmail.com
Recombinant vaccine technology employs yeast or bacterial cells to made many copies of a particular viral or bacterial protein or sometimes a small part of the protein but mRNA vaccines bypass this step. They are chemically synthesized without the need for cells or pathogens, making the production process simpler. While the concept of mRNA vaccines may seem simple, the technology is rather sophisticated. RNA is a notoriously fragile molecule. Delivering mRNA successfully to cells inside our bodies and ensuring that enzymes within our cells do not degrade it are key challenges in vaccine development ${ }^{7}$.

Since mRNA vaccines are developed in a synthetic manner, there is no limitation to the number of vaccines that can be manufacture but these vaccines are expensive and they need to be stored at an extremely cold temperature ${ }^{8}$.

Unlike many other vaccines that contain an infectious pathogen or a part of it, viral vector vaccines use a harmless virus to deliver a piece of genetic code to our cells, allowing them to make a pathogen's protein. This trains our immune system to react to future infections. The Oxford-AstraZeneca COVID-19 vaccine uses a chimpanzee common cold viral vector known as ChAdOx1, which delivers the code that allows our cells to make the SARS-CoV-2 spike protein. Then our cells present the spike protein on the cell surface, prompting our immune system to make antibodies and mount $\mathrm{T}$ cell responses ${ }^{9}$.

On December 11, 2020, the U.S. Food and Drug Administration issued the first emergency use authorisation to Pfizer-BioNTech's vaccine for its use in the U.S. among individuals 16 years of age and older. Soon it was rolled out in the United Kingdom, Canada and several mainland European countries. On December 31, the World Health Organisation (WHO) listed the COVID-19 mRNA vaccine for emergency use, making the Pfizer-BioNTech vaccine the first to receive emergency validation from WHO since the outbreak began a year ago ${ }^{8}$.

Till date few approved vaccines are Pfizer-BioNTech's mRNA-based vaccine, Moderna's mRNA-based vaccine, AstraZeneca's viral vector vaccine, Gamaleya Research Institute's Sputnik V viral vector vaccine, Sinovac's Inactivated vaccine and Bharat Biotech's, Covaxin Inactivated vaccine ${ }^{10}$. 
Two doses of above mentioned mRNA-based vaccines have now shown remarkable effectiveness of about $95 \%$ in preventing COVID-19 disease in adults. For Pfizer-BioNTech COVID-19 vaccine, the interval is 21 days and for Moderna COVID-19 vaccine, the interval is 28 days between the first and second dose $\mathrm{e}^{11}$. Both of these mRNA-based vaccines are available as multi dose vial (up to 6 doses per vial in Pfizer-BioNTech and upto 10 doses per vial in Moderna). Pfizer-BioNTech vaccine must need to be mixed with diluent before administration but Moderna vaccine need no to mix with a diluents ${ }^{12,13}$. These vaccines should be preserved at extremely cold temperature (Pfizer's vaccine at -70 degrees Celsius and Moderna's vaccine at $-25^{\circ} \mathrm{C}$ and $15^{\circ} \mathrm{C}$ temperature $)^{8}$.

Oxford-AstraZeneca also introduces multidose vials (upto 10 doses per vial) but have a shelf life of 6 months when stored at $2^{\circ} \mathrm{C}$ to $8^{\circ} \mathrm{C}$. After first dose withdrawal, the multidose vial should be used as soon as possible and within 6 hours. The multidose vials can be stored at $2{ }^{\circ} \mathrm{C}$ to $25^{\circ} \mathrm{C}$ during their in-use period ${ }^{14}$. Vaccine should be administered intramuscularly and vaccination course consists of two separate doses between 4 and 12 weeks aprt ${ }^{15}$. This vaccine has got $70.4 \%$ efficacy $^{16}$.

Due to easy storage, delivery facility and low cost; COVID-19 vaccination has been started in Bangladesh from $7^{\text {th }}$ February 2021 with Oxford-AstraZeneca vaccine. We are expecting a COVID-19 free world very soon, inshAllah.

\section{References :}

1. World Health Organization. Pneumonia of unknown cause-China 2020 -https://www .who .int/ csr/ don/05 - january-2020-pneum onia-of-unkown-cause-china/en/.

2. "Bangladesh confirms its first three cases of coronavirus". Reuters. 8 March 2020. Archived from the original on 27 March 2020 Retrieved 27 March 2020.

3. Available from- https://www.worldometers.info/coronavirus/Last updated: February 06, 2021, 05:00 GMT

4. Available from-https://www.who.int/emergencies/diseases/novelcoronavirus-019/covid-19-vaccines.

5. Available from- https://www.who.int/news-room/feature-stories/ detail/manufacturing-afety-and-quality-control.

6. Available from- https://www.who.int/news-room/feature-stories/ detail/the-race-for-a-ovid-19-vaccine-explained.

7. Hewings-Martin Y. December 18, 2020https://www.medicalnews today.com/articles/covid-19-how-do-mRNA vaccines-work.

8. Ahuja A. Coronavirus Outbreak Explained: What Is mRNA COVID-19 Vaccine Technology Used By Pfizer-BioNTech And Moderna? January 15, 2021 5:25 PM (https://swachhindia .ndtv.com/coronavirus-outbreak-explained-what-is-mrna-covid19 -vaccine-technology-used-by-pfizer-biontech-and-moderna$55342 /$.
9. Hewings-Martin Y. January 15, 2021 https://www.medical news today.com/articles/covid-19-how-do-viral-vector-vaccines-work.

10. Available from-https://www.raps.org/news-and-articles/news-arti cles/2020/3/covid-19-vaccine-tracker.

11. Stephen M, Marks P. FDA Statement on Following the Authorized Dosing Schedules for COVID-19 Vaccines. Press release, January 04, 2021 -https://www.fda.gov/news-events/pressannouncements/fda-statement-following-authorized-dosingschedules-covid-19-vaccines\#: : text $=$ The $\% 20$ available $\% 20$ data $\% 20$ continue $\% 20$ to, the $\% 20$ first $\% 20$ and $\% 20$ second $\% 20$ dose.

12. Available from- https://www.cdc.gov/vaccines/covid-19/info-byproduct/pfizer/index.html.

13. Available from-https://www.cdc.gov/vaccines/covid-19/info-byproduct/moderna/index.html.

14. Available from-https://www.sps.nhs.uk/articles/astrazeneca-covid19-vaccine-formulation-information/

15. Bischler A. Dosing information for AstraZeneca COVID-19 Vaccine. Published 13 January 2021 (https://www.sps.nhs. uk/articles/dosing-information-for-astrazeneca-covid-19-vaccine/

16. Knol MD, Wonodi C. Oxford-AstraZeneca COVID-19 vaccine efficacy Published Online December 8, 2020. Lancet;397 (10269). https://doi.org/10.1016/ S0140-6736(20)32623-4. 